\title{
LA FORET DU PORTUGAL CENTRAL: UN PAYSAGE EN MUTATION*
}

\author{
António Campar de Almeida**
}

\begin{abstract}
RESUMO
A actual paisagem florestal da Região Centro pode ser caracterizada por uma variação litoralinterior segundo seis faixas mais ou menos irregulares e com diversas composições de árvores, tais como pinheiros bravos quase em absoluto, estes em mistura com eucaliptos ou com carvalhos. eucaliptos quase exclusivos e carvalhos dominantes.

Durante as últimas décadas verificaram-se notórias modificações em algumas das sub-regiões. As matas de eucaliptos aumentaram quase por todo o lado, normalmente à custa dos pinhais. Os carvalhais têm também aumentado, especialmente no interior Norte.

As causas destas modificações devem-se não só à forte procura de madeira de eucalipto por parte das fábricas de pasta de papel, mas também, no que respeita aos carvalhais do interior. ao abandono - sucessivo de terrenos de cultura por uma população rural cada vez mais escassa e envelhecida.

Os incêndios florestais desempenham um papel fundamental na composição paisagística da Região Centro. Assiste-se ao desenvolvimento de grandes manchas de árvores com a mesma idade, bem como a áreas cada vez mais importantes com matos e também à proliferação de espécies exóticas muito competitivas e invasoras.
\end{abstract}

Palavras-chave: Floresta. Região Centro. Paisagem. Incêndios

\section{RÉSUMÉ}

Le paysage forestier actuel de la Région Centre du Portugal peut être caracterisé par une variation littoral-intérieur selon six bandes plus ou moins irrégulières, avec différents types d'arbres - des pins maritimes purs, en mélange avec des eucalyptus ou avec des chênes, des eucalyptus purs et des chênes dominants

Pendant les dernières décades on a constaté des modifications assez significatives dans quelquesunes des sous-régions. Les bois d'eucalyptus ont augmenté presque partout, normalement aux dépens des pinèdes. Les chênais ont aussi augmenté, notamment à l'intérieur Nord.

Les causes de ces modifications relèvent de la forte demande du bois d'eucalyptus par les usines de pâte à papier, mais aussi, pour les chênais de l'intérieur, à l'abandon successif de terrains de culture, de la part d'une population rurale de plus en plus rare et vieillie.

Les incendies forestiers jouent un rôle fondamental dans la composition paysagère de la Région Centre. On voit le développement de grandes tâches d'arbres avec le même âge, des aires de plus en plus importantes avec des broussailles et l'invasion d'espèces exotiques très compétitives.

Mots-clés: Forêt. Région Centre. Paysage. Incendies

\begin{abstract}
A BSTRACT
The Central Region's forest landscape presents a littoral-inland variation according to six irregular belts with different tree compositions: maritime pine only, pine mixed both with eucalyptus and with oak trees, eucalyptus only and oak trees.

Clear changes have been registered during the last decades in some of the subregions. Eucalyptus woods spread almost everywhere, normally at the expense of pine woods. Oak forests have increased especially in the North inland.
\end{abstract}

\footnotetext{
*O texto corresponde à comunicação apresentada no Colóquio "El bosque mediterráneo: qué herencia y qué porvenir?", em Madrid, na Casa Velásquez, em Fevereiro de 2000.

${ }^{* *}$ Centro de Estudos Geográficos. Faculdade de Letras. Universidade de Coimbra.
} 
The causes of these changes are not only due to the intense demand of eucalyptus timber by papermills, but also, in what concerns inland oak forests, to the progressive abandonment of arable lands by an increasingly rare and aged rural population.

The forest fires play an important part in the landscape composition of the Central Region. Great patches of same aged trees, significant surfaces of heathlands as well as of very competitive and invasive exotic species have developed.

Key-words: Forest. Central Region. Landscape. Forest fires

Pendant ces derniers siècles, la région Centre du Portugal a souffert une évolution qui ressemble à celle du reste du pays en ce qui concerne la superficie forestière. Le recul de la forêt a été constant, et il est accompagné depuis peu par une reconversion de sa composition floristique. Les défrichements effectués par une population toujours croissante ont fait peu à peu disparaitre les chênaies primitives ; à partir du XIXe siècle, ce processus a été accompagné par la diminution des châtaigneraies, décimées par la maladie de l'encre (DEVY-VARETA, 1993).

Tout au long de ce même siècle, bien que d'un mode irrégulier, des plantations de pin maritime sont installées dans quelques régions du littoral. C'est à ce moment-là que l'on commence à reconnaître l'importance de la forêt comme une ressource stratégique du pays. Le pin sera plus tard, à partir du début du XXe, l'essence préférée des reboisements effectivement réalisés par les services forestiers de l'Etat, par l'intermédiaire de divers programmes de plantations, non seulement dans les dunes littorales, comme aussi dans les communaux (baldios) montagnards. La préférence pour cette espèce est dû à sa rapide croissance, et surtout au coût minime des semis (OLIVEIRA e ALVES, 1993). A l'échelle du pays, la superficie du pin a augmenté jusqu'au début des années 80 et recule depuis cette date (AA. VV., 1998).

Ce recul est dû à l'expansion de l'eucalyptus, en croissance continue depuis la seconde moitié du XXe, et provoquée par l'installation de plusieurs usines de pâte à papier.

\section{LA COUVERTURE FORESTIERE DANS LA REGION CENTRE}

Lorsque l'on parcourt aujourd'hui la région Centre (Fig. 1), on vérifie facilement que la composition de la forêt se modifie beaucoup plus lorsque l'on se déplace du littoral vers l'intérieur, qu'en allant du Nord vers le Sud. $\mathrm{Ce}$ fait vient, d'ailleurs, plus ou moins reproduire la distribution des grands domaines arborés antérieurs à l'anthropisation de la forêt, c'est à dire, quand les chênes étaient les espèces dominantes.

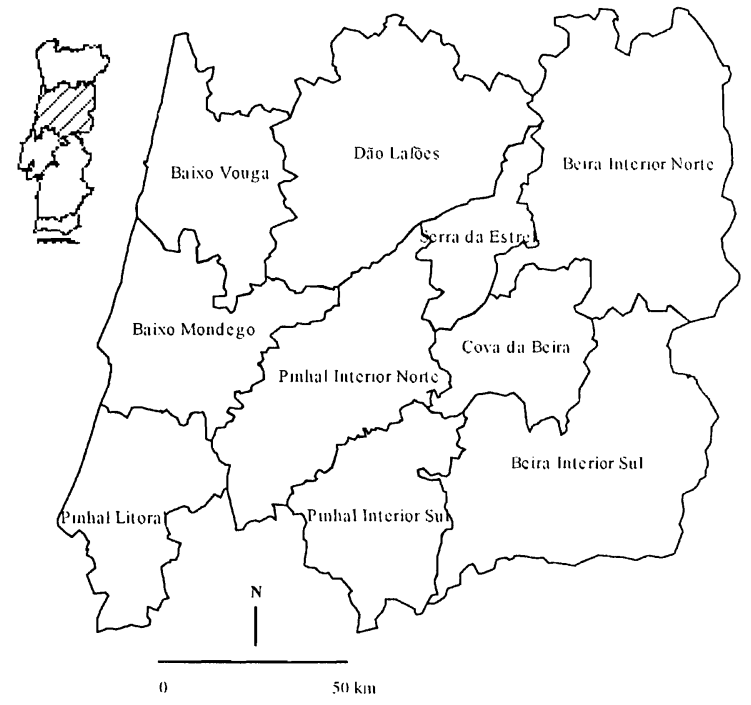

Fig. 1 - Région Centre du Portugal et ses sous-régions.

Près des côtes, sur les dunes, on trouve de grandes étendues de pins maritimes (Pinus pinaster Aiton), où l'espèce est pratiquement exclusive (Fig. 2). Il s'agit de pinèdes de reboisement, dont le sous-bois intègre des acacias, qui ont permis la fixation des dunes, et des fruticées psammophiles.

Dans le reste de la bordure (Orla) Méso-cénozoïque, où se succèdent plusieurs plates-formes sableuses recoupées par des fleuves, les masses forestières sont constituées par le pin maritime encore dominant et l'eucalyptus (Eucalyptus globulus Labill.). Dans les sous-bois, les chênes-lièges (Quercus suber L.), les chênes portugais (Quercus faginea Lam.) et les arbousiers (Arbutus unedo L.), au-dessus des ajoncs (Ulex spp.) et des bruyères (Erica spp. et Calluna vulgaris L.), essaient de reconquérir leur importance dans la forêt. Quand le calcaire affleure et forme des collines ou des monts, la couverture forestière est plus éparse. Elle est souvent constituée par des chênes portugais et des chênes verts (Quercus rotundifolia Lam.). Des petits massifs de pin maritime, de pin pignon (Pinus pinea L.) et d'eucalyptus sont aussi fréquents 
Les accidents topographiques qui correspondent aux montagnes occidentales du Massif Ancien, généralement schisteuses, provoquent un changement: l'eucalyptus devient l'essence presque exclusive, en occupant plus de $80 \%$ de la surface. Cette bande atteint une largeur maximale dans la partie occidentale de la plate-forme du Mondego (Fig. 2). Le sous-bois, quand il existe, est constitué par des bruyères, ajoncs et Chamaespartium tridentatum (L.) P. GIBBS, sur les schistes, ou des genêts (Cytisus spp. et autres) sur les granites.

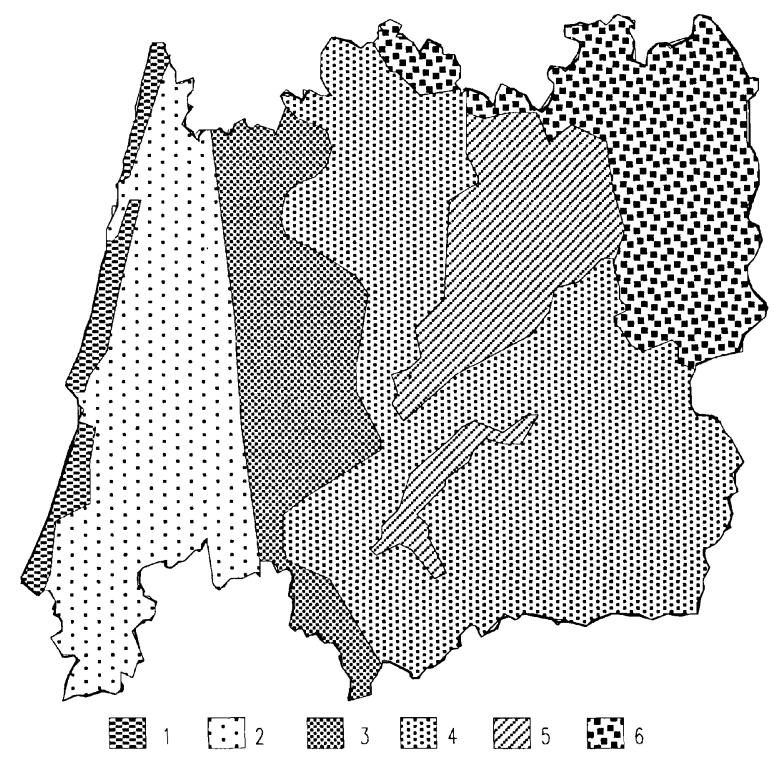

Fig. 2 - Bandes d'arborisation dans la Région Centre. 1- Pinède des dunes ; 2- Pins et eucalyptus dans l'Orle; 3- Eucalyptus; 4- Pins et eucalyptus de l'intérieur ; 5- Pinède de l'intérieur; 6- Chênaies
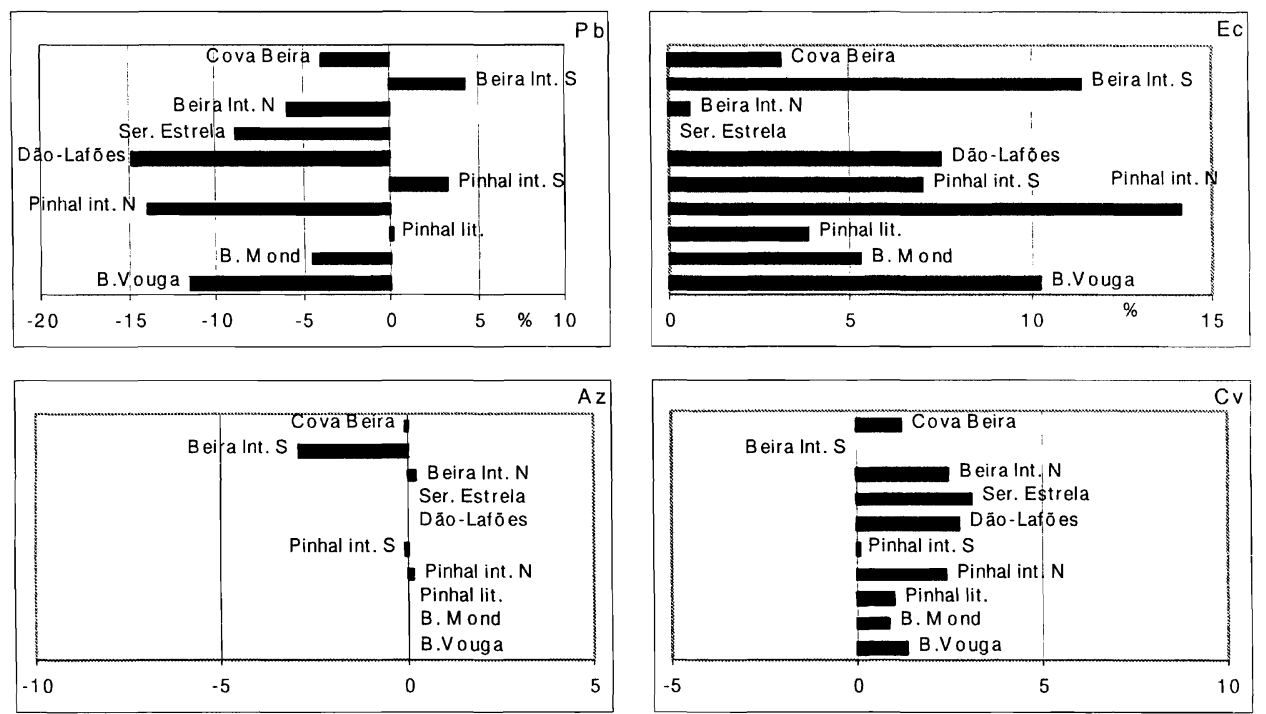

Fig. 3 - Changements spatiaux (\%) des essences forestiers pin, eucalyptus, chêne vert et chêne. empêchent leur expansion primitive. genêts ou de bruyères dans la strate inférieure. pées. La couverture arborée est très irrégulière.

\section{LES CHANGEMENTS RECENTS} que plus modestement, sauf dans la Beira Interior Sul.

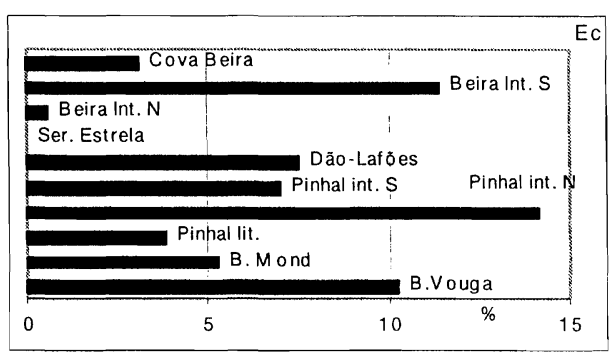

A l'Est, dans les régions attenantes, on retrouve le mélange de pin maritime et eucalyptus, avec l'intromission d'un important massif de pin pignon dans le centre de la plate-forme du Mondego. Dans la partie sud, apparaissent des bois de chênes verts et tauzins (Quercus pyrenaica Willd.), alors qu'au nord, surgissent les chênes rouvres (Quercus robur L.) et tauzins. Ces derniers, par ailleurs, se développent fréquemment à l'ombre des pins ou des eucalyptus et ce sont uniquement les coupes qui

Plus à l'intérieur et en altitude, les eucalyptus perdent leur importance. Dans un premier secteur, ce sont les pins maritimes qui dominent à nouveau, mais ces pinèdes se distinguent de celles des régions côtières, puisqu'ici, à l'intérieur, le sous-bois est composé de chênes tauzins, de

Finalement, le plateau nord-est, plus froid et sec, est dominé par le tauzin, et l'on peut voir ici et là quelques plantations de pins maritimes relativement bien dévelop-

En se basant sur les inventaires forestiers de 1974 et 1995 élaborés par la Direction Générale des Forêts, on peut saisir les changements spatiaux les plus significatifs, qui ont touché trois essences forestières dans la région Centre. Le pin maritime a souffert une diminution générale, à l'exception de la Beira Interior Sul et du Pinhal Interior Sul (Fig. 3). En contrepartie, l'eucalyptus a augmenté dans toutes les sous-régions, la plupart des fois en remplaçant le pin maritime. Le chêne (l'espèce n'est pas différenciée dans les inventaires) a aussi vu sa surface augmentée, bien 
On arrive aux mêmes conclusions en comparant les pourcentages des espèces par sous-région, entre les deux inventaires (Fig. 4). Dans presque toutes ces unités, le pin perd de l'importance en faveur de l'eucalyptus; mais
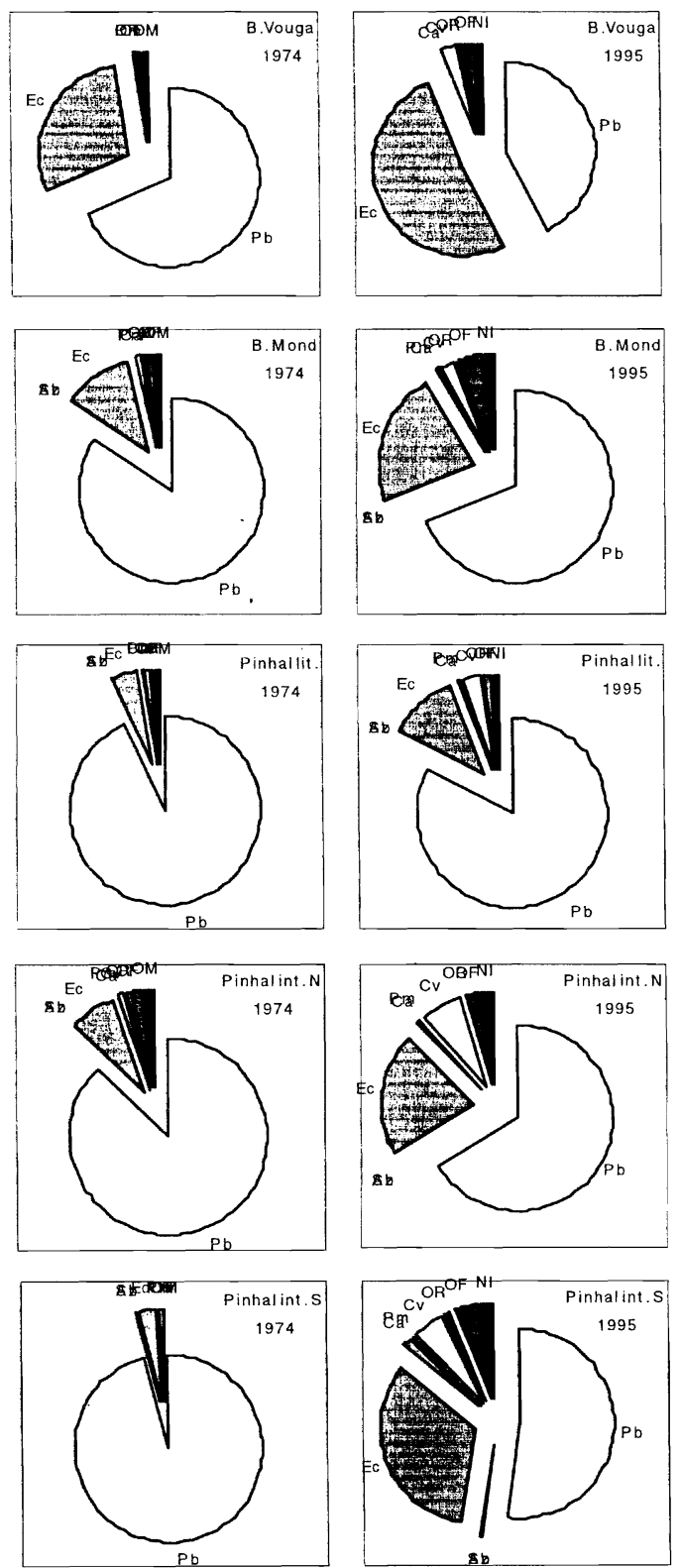

c'est en faveur du chêne que le pin diminue dans la Serra da Estrela et la Beira Interior Norte. Par contre, dans la Beira Interior Sul, l'eucalyptus augmente au détriment du chêne vert.
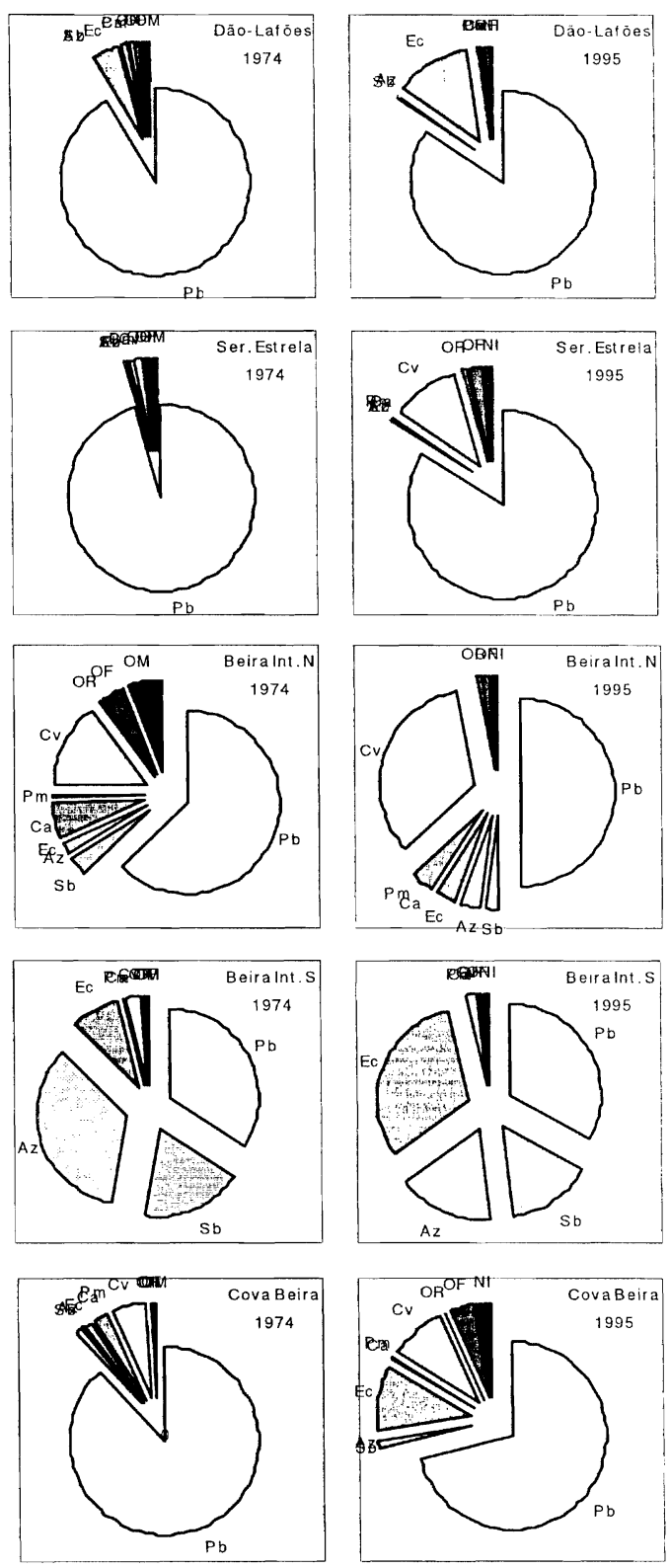

Fig. 4 - Variation des essences forestiers entre 1974 et 1995, dans les sous-régions de la Région Centre 
Malgré une légère augmentation du taux de boisement dans la région, prés de $3 \%$, les valeurs absolues dénoncent une diminution significative du pin et une baisse modérée dans le cas du chêne vert. Ces valeurs montrent aussi la forte expansion de l'eucalyptus, et une augmentation plus modeste dans le cas du chêne (Table I).

Table I - Variation de la superficie de quelques espèces entre 1974 e 1995.

\begin{tabular}{|l|c|l|c|}
\hline Espèce & Diminution (ha) & \multicolumn{1}{|c|}{ Espèce } & Augmentation (ha) \\
\hline Pin maritime & -131210 & Eucalyptus & 162045 \\
Chêne vert & -9577 & Chênes & 36243 \\
Châtaignier & -2761 & Chêne liège & 2455 \\
Pin pignon & -1990 & & \\
\hline
\end{tabular}

\section{LES FACTEURS DES CHANGEMENTS}

Dans la région Centre, les changements de la forêt sont surtout le réflexe des modifications socio-économiques vérifiées depuis les dernières décennies. Le dépeuplement des campagnes, le vieillissement de la population rurale locale, la diminution du secteur primaire, la forte valorisation du bois d'eucalyptus, les incendies forestiers et les propres mesures européennes incitant à l'abandon de quelques produits agricoles constituent les facteurs qui ont le plus contribué à la modification de la forêt du Centre. Pour toutes ces raisons, le paysage a acquis un nouvel aspect.

La diminution de la population rurale est aussi accompagnée de changements dans sa propre structure. On vérifie un vieillissement presque généralisé, aussi bien à la base qu'au sommet de la pyramide des âges, c'est à dire que le nombre de jeunes diminue alors que celui des personnes âgées augmente. Entre 1970 et 1991 (Fig. 5), la relation entre la population de moins de 15 ans et celle de - plus de 64 ans montre l'impossibilité de la substitution des personnes âgées dans un proche avenir, et ceci dans six des sous-régions du Centre. Donc, la rénovation des bénéficiaires et des intervenants potentiels est fortement comprise dans ce territoire.

Parallèlement, on assiste à une transférence - de 30 à $50 \%$ - de la population active du secteur primaire lié à l'agriculture et à la sylviculture vers les autres secteurs d'activité (Fig. 6). Les sous-régions de l'intérieur, qui en 1970 présentaient les valeurs de 60 à $70 \%$ (à l'exception de la Cova da Beira, grâce à la zone industrielle de Covilhã) sont celles qui ont vu la plus forte diminution de l'emploi dans le secteur primaire, compris de nos jours entre 10 et $30 \%$. Ces valeurs se rapprochent de celles des sous-régions plus industrialisées du littoral.

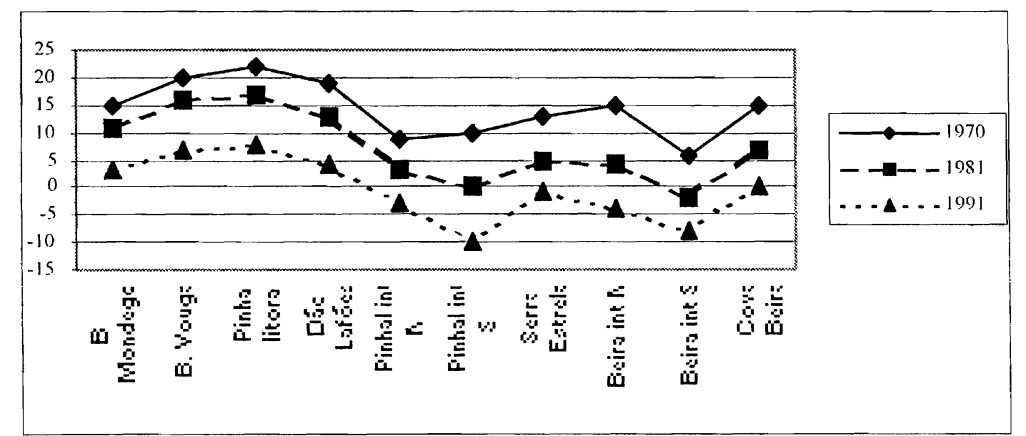

Fig. 5 - Différence entre le pourcentage des gens avec moins de 15 ans et plus de 64 ans.

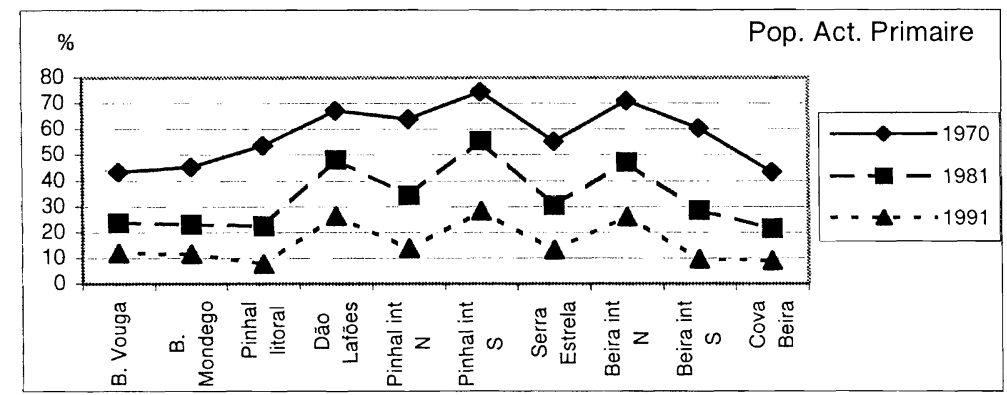

Fig. 6 - Évolution de la population active primaire entre 1970 et 1991. 
L'entrée en fonctionement des usines de pâte à papier a provoqué une forte demande de bois d'eucalyptus, principale matière première de la fibre cellulosique. Son prix a rapidement atteint celui du pin maritime, qui était le plus grand fournisseur de la filière industrielle forestière. Rappelons qu'un eucalyptus atteint en 10 ans la même hauteur qu'un pin en 40-50 ans (GOMES e OLIVEIRA, 1990). Il est donc évident que de nombreux producteurs ont choisi cette esCence exotique, qui offre à court terme de meilleurs revenus. C'est surtout à partir des années 1970 , losque les conditions écologiques lui sont favorables, que la plantation d'eucalyptus devient dominante.

On peut constater que, pendant les décennies antérieures à 1970 , le reboisement a été surtout fait à base d'un résineux, le pin maritime. Depuis, il est effectué à l'aide d'eucalyptus, ou de pin dans les régions antérieures.

L'évolution du reboisement a donc conduit à l'installation de massifs forestiers très étendus et monospécifiques, ou composés à peine de deux espèces, eucalyptus et pin, qui en plus sont extrémement combustibles. Le résultat est l'occurrence d'un grand nombre d'incendies, détruisant des milliers d'hectares de forêt par an, surtout dans les zones accidentées où le combat contre le feu est plus difficile (Fig. 7). On vérifie que l'incidence du feu est plus fréquente dans les sous-régions de Dão-Lafões et Beira Interior Norte et, à moindre dégré d'importance, dans celles qui intègrent et entourent la montagne de Estrela, c'est à dire, où l'accès est plus difficile et la population moins dense. Pendant ces quinze dernières années, les surfaces incendiées dans Dão-Lafões et Beira Interior Norte correspondent à presque la totalité de la superficie forestière de ces sous-régions (Fig. 8).
Les subventions européennes incitant à l'abandon de productions agricoles, comme par exemple la vigne, ont eu deux conséquences: (1) la reconversion des terrains agricoles en surfaces forestières, composées d'espèces au bois dur (chêne américain, noyer, cyprés du Buçaco, châtaignier, platanes, etc); (2) l'extension des jachères, permettant de développement de la végétation spontanée, qui, à long terme, peut évoluer vers une formation arborée.

\section{LES MUTATIONS PAYSAGERES}

Le choix de nouvelles espèces forestières, les changements sociaux du monde rural et les conséquences directes et indirectes des incendies ont provoqué de profondes modifications dans la physionomie et la composition de la forêt de la région Centre.

Dans la moitié occidentale, on peut parler d'une authentique «eucalyptisation» du territoire. Dans la plaine littorale, l'alternance des bois d'eucalyptus et des pinèdes, parfois remplacées par quelques parcelles de divers conifères ou feuillus, s'oppose en quelque sorte à la monotonie des pinèdes monospécifiques côtières.

La monotonie paysagère se manifeste aussi dans les montagnes schisteuses du littoral, que se soit par les surfaces continues plantées d'eucalyptus, ou par la rareté de l'occupation humaine. Les uniques touches de diversité se traduisent par des parcelles de différentes couleurs, redevables aux aucalyptus de diverses tranches d'âge, à quelques rares pinèdes et aux terres cultivées près des villages encore peuplés.

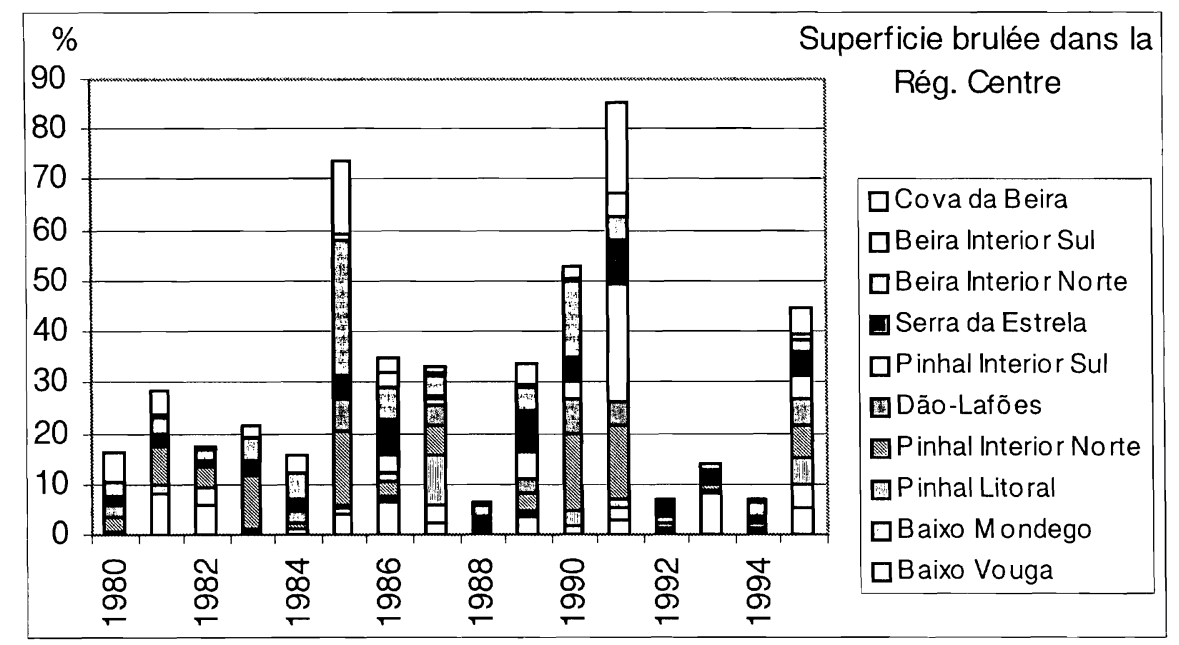

Fig. 7 - Superficie brulée dans la Région Centre entre 1980 et 1995 (pourcentage de l'aire forestière à chaque sous-région). 
La fréquence des incendies a facilité la prolifération des espèces exotiques, fortement compétitives et pyrophites, comme les acacias (surtout Acacia dealbata Link) et les ailanthes (Ailanthus altissima (Miller) Swingle). Ces exotiques se disséminent à partir des marges des fleuves ou du bord des routes. Leur expression spatiale est encore faible, mais leur diffusion augmente, vu leur difficile erradication.

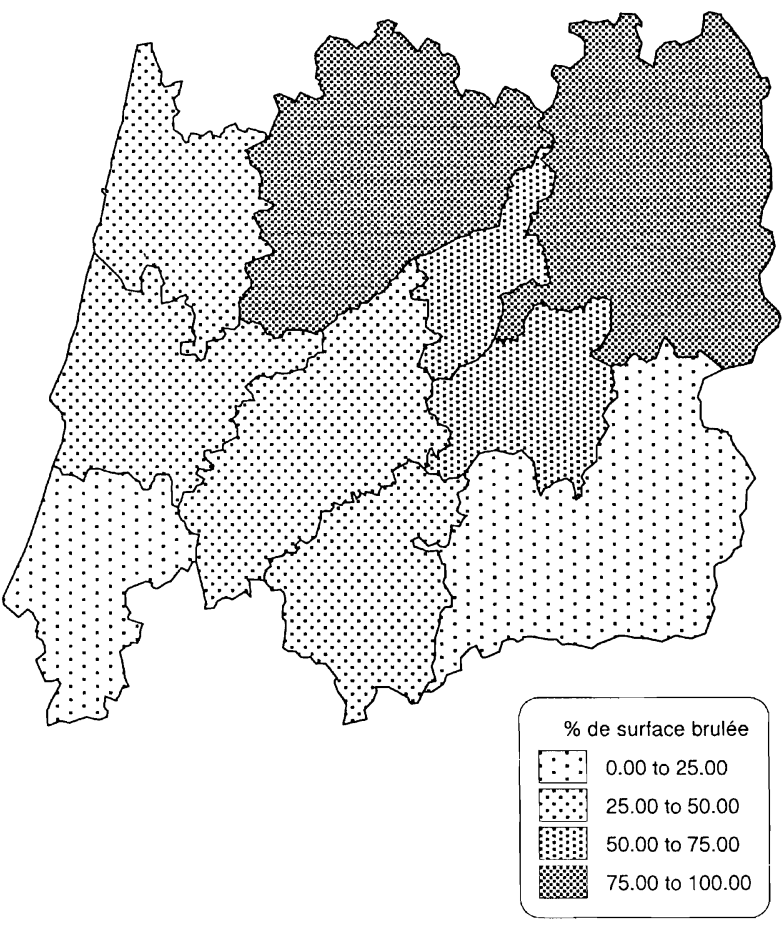

Fig. 8 - Pourcentage accumulée de surface brulée entre 1980 et 1995

Dans les monts calcaires, on assiste à un dépeuplement progressif et à l'abandon des terres agricoles, qui sont rapidement occupées par le chêne kermès (Quercus coccifera L.), l'une des espèces arbustives des premières étapes de l'évolution vers la chênaie de chêne portugais. Quand le sol est plus profond, on plante aussi des eucalyptus. Les terrains plus marneux, cultivés il y a peu de temps encore, ont fait l'object de plantations de cyprès du Buçaco (Cupressus lusitanica Miller). La plupart ont réussi, mais se limitent à peine à quelques parcelles isolées (ALMEIDA et al., 1999).
Dans la moitié orientale de la région, l'évolution peut se faire dans le sens d'un rapprochement de la végétation naturelle, à l'exception de la Beira Interior Sul où l'eucalyptus a pris une certaine importance dans la superficie forestière au détriment du chêne vert.

La diminution de l'agriculture et de l'élevage a libéré des surfaces pour la forêt, mais les incendies ont limité son expansion. Les vastes étendues de genêts au nord de la cordillère centrale, et de cistes au sud sont le témoin du fréquent passage du feu. Les pins, à la croissance ici plus lente, et les chênes, au développement naturellement lent, surgissent au milieu de ces arbustes et reformeront peutêtre la couverture forestière. Les terres abandonnées sont immédiatement envahis par les genêts, puis par les chênes, en général le tauzin. Quelques parcelles sont plantées de châtaigniers (Castanea sativa Miller), puisque la demande de la châtaigne est assez forte sur les marchés, malgré le risque toujours présent de la maladie de l'encre.

Dans la Serra da Estrela, où les altitudes sont supérieures, on a éssayé la plantation d'espèces forestières d'altitude avec succés, comme le pin sylvestre (Pinus sylvestris L.) et le bouleau (Betula celtiherica Rothm. et Vasc.), qui autrefois couvraient ce massif.

\section{RÉFÉRENCES BIBLIOGRAPHIQUES:}

AA. VV. (1998) - Livro verde sobre a cooperação ensino superior-empresa. O sector florestal. CESE - Conselho para a Cooperação Ensino Superior-Empresa. 169 p.

Almeida, A.; Nunes, A.; Amante, T.; Martinho, C. e Sillva. B. (1999) - "Análise ecológica da parsagem do Baixo Mondego: uma abordagem inicial". Encontros de Geomorfologia. Coimbra, pp. 173-179.

DeVy-VARETA, Nicole (1993) - A floresta no espaço e no tempo em Portugal. A arborização da Serra da Cabreira (1919. 1975). Fac. Letras Univ. Porto, 459 p. (tese de doutoramento)

Gomes, A. A. e Oliveira, J. S. (1990) - "O eucalipto em Portugal, na óptica de um modelo de desenvolvimento sustentável". In O eucalipto em Portugal. Na óptica de um desenvolvimento sustentável. Inst. Nac. Ambiente. pp. 19102.

Oliveira, A. Carvalho e Alves. A. Monteiro (1993) - "Longterm afforestation programmes in Portugal". VOLZ, K.-R. e WEBER, N. (ed.) - Agriculture. Afforestation of agricultural land. Comm. European Communities, pp. 13-22. 